\title{
Evidence of Immune disorder served as the main pathogenesis in SSNHL: elevated serum concentration of MMP-9 following treatment was associated with favorable outcomes in patients
}

\author{
Tao Lu ${ }^{1}$, Hong Chen ${ }^{1}$, Jiaqiu Dai ${ }^{1}$, Siji Wang ${ }^{1}$, Ziqi Chen ${ }^{1}$, and Houyong Kang ${ }^{1}$ \\ ${ }^{1}$ The first affiliated hospital of Chongqing Medical University
}

June 22, 2020

\begin{abstract}
Objectives: Sudden sensorineural hearing loss (SSNHL) is an emergency disease with undefined pathogenesis in the otolaryngology department. In our previous study, we found patients with SSNHL had lower serum concentration of Matrix metalloprotease 9 (MMP-9) than healthy controls, and the result was accordant with auto-immune diseases. This study aimed to reveal the correlation between changes in serum MMP-9 concentration following treatment with the outcomes of patients and to provide further evidence that immune disorder was the main pathogenesis of SSNHL. Design, setting, and participants: Fifty-two patients with SSNHL, hospitalized in The First Affiliated Hospital of Chongqing Medical University from March 2019 to August 2019, were enrolled. The serum concentration of MMP-9 was detected by enzyme-linked immunosorbent assay (ELISA). Main outcome measure: The mean concentration of MMP-9 before treatment was compared with the post-treatment concentration by the Mann-Whitney U test. The correlations between favorable outcomes of patients and clinical characteristics were measured with the Chi-squared test and binary multiple logistic regression analysis. Results: In treatment responders, mean serum concentration was elevated from $106.85 \pm 41.40 \mathrm{ng} / \mathrm{ml}$ to $144.03 \pm 37.65 \mathrm{ng} / \mathrm{ml}$ following treatment $\left(\mathrm{P}_{\mathfrak{j}} 0.001\right)$, while in non-responders it decreased from $132.09+-59.21 \mathrm{ng} / \mathrm{ml}$ to $106.82+-49.93 \mathrm{ng} / \mathrm{ml}(\mathrm{P}=0.142)$. Changes in MMP-9 concentration was the only factor associated with favorable outcomes $(\mathrm{P}=0.008, \mathrm{OR}=5.13,95 \% \mathrm{CI}$ : 1.53-17.28). Conclusions: Elevated MMP-9 concentration is a potential prognosis biomarker in patients with SSNHL. These findings are in line with auto-immune diseases and indicate immune disorder is mainly pathogenesis in SSNHL. Keywords Sudden sensorineural hearing loss; Matrix metalloprotease 9; Immune disorder; prognosis; Enzyme-linked immunosorbent assay
\end{abstract}

\section{Introduction}

Sudden sensorineural hearing loss (SSNHL) is defined as an acute sensorineural hearing loss occurring within 72 hours and characterized by a hearing loss of $30 \mathrm{~dB}$ or greater over in at least three contiguous audiometric frequencies [1]. The incidence of SSNHL varies from 5 to 30 per 100000 people [2,3], and the pathogenesis of SSNHL remains unclear. Autoimmune disorders, viral infection, and microvascular injury have been described as one of the etiologies of SSNHL, but none of them are defined definitely $[4,5]$.

MMP-9 is one of the Matrix metalloproteinases (MMPs), which is a large family of zinc-containing calciumdependent endopeptidases. MMP-9 is mainly distributed in the extracellular matrix and has a function to degrade multiple extracellular components, such as collagen and elastin [6]. Several studies have shown MMP-9 is involved in the pathogenesis of diseases including tumor, cardiovascular, and autoimmune disease. Intriguingly, the results in different diseases were in contrast. The mean serum concentration of MMP-9 in patients with tumors or cardiovascular diseases was higher than in healthy controls, whereas MMP-9 level in patients with autoimmune diseases such as Systemic Lupus Erythematosus (SLE) was lower than in healthy controls [7-11]. In our previous study, we delineated that the serum level of MMP-9 in patients with SSNHL was lower than in healthy controls, and the result was in line with autoimmune diseases 
rather than tumors or cardiovascular diseases [12]. Therefore, to further provide evidence that immune disorder was the main pathogenesis of SSNHL, we collected serum from 52 patients with SSNHL prior to and following treatment with detecting the serum concentration of MMP-9 by ELISA. We hypothesized the mean serum concentration of MMP-9 following treatment elevated in treatment responders and the elevation was associated with favorable outcomes of patients with SSNHL.

\section{Materials and methods}

\subsection{Audiometric Evaluation}

All the patients received pure-tone audiometry (PTA) before treatment - following a one-week and two-week treatment. Mean pure-tone audiograms was defined as the average of air-conduction threshold measured at $500,1000,2000,4000 \mathrm{HZ}$ and was employed to describe outcomes of patients.

Four types of audiograms were identified according to the initial pattern of hearing loss with PTA: ascending (the average threshold of 0.25 to $0.5 \mathrm{kHz}$ was $20 \mathrm{~dB}$ higher than the average threshold of 4 to $8 \mathrm{kHz}$.), descending (the average threshold of 4 to $8 \mathrm{kHz}$ was $20 \mathrm{~dB}$ higher than the average threshold of 0.25 to 0.5 $\mathrm{kHz}$ ), horizontal (Less than $20 \mathrm{~dB}$ threshold difference observed across all frequency and the mean hearing threshold of $0.5,1,2$ and $4 \mathrm{KHZ}$ was no more than $80 \mathrm{~dB}$ ), profound (similar audiogram shapes to the horizontal group, whereas the average threshold of $0.5,1,2$ and $4 \mathrm{KHZ}$ was no less than $81 \mathrm{~dB}$ ).

As indicated in Chinese clinical practice guidelines of SSNHL [13], patients were stratified into four groups based on their responses to treatment (criteria were shown in Table. I): Nonrecovery (NR), partial improvement (PI), marked improvement (MI) and complete recovery (CR). Patients in the NR group were defined as the treatment non-responders, while patients in the other three groups were defined as the treatment responders.

\subsection{Patients and serum samples}

Fifty-two patients with SSNHL, admitted to [removed for blind peer review] from March 2019 to August 2019, were enrolled in this study. Eligible patients for this study met the following criteria: 1) had sensorineural hearing loss of $30 \mathrm{~dB}$ or greater over in at least three contiguous audiometric frequencies within 72 hours; 2) had no history of retrocochlear disease; 3) received treatment in 3 days after onset and had no treatment in other hospitals. Patients were excluded if they had the following diseases: 1) hypertension; 2) diabetes mellitus; 3) autoimmune disease; 4) cancer; 5) any other contraindications for glucocorticoid. Patients were discharged if they met the criteria of $\mathrm{CR}$ or had a two-week time of hospitalization. Blood samples were collected before and following treatment and serums were separated after centrifugation and stored at -80 immediately.

\subsection{Treatment Protocol}

All the patients received following therapy: 1) oral prednisolone at a dose of $1 \mathrm{mg} / \mathrm{kg}$ per day in the first week of therapy; 2) extract of ginkgo biloba leaves intravenous injection (i.v) at a dose of $20 \mathrm{ml}$ per day; 3) oral mecobalamin tablets at a dose of $0.5 \mathrm{mg}$ three times a day; 4 ) batroxobin i.v at a dose of $10 \mathrm{BU}$ for the first time and 5BU every other day; 5) intratympanic corticosteroids at a dose of 40mg every other day in the second week if patients did not meet the criteria of CR following treatment with oral prednisolone.

\subsection{ELISA}

Elisa kits for MMP-9 were purchased from RD System Inc(Catalog No: DM-900). Serum samples were thawed under room temperature for 10 minutes, diluted at 1:100, and then transferred to polypropylene tubes. The wells added with 100ul of Assay Diluent were incubated for 2 hours at room temperature on a horizontal orbital microplate shaker with 100ul of standard or samples. After washing with Wash Buffer for 4 times, 200ul of Human MMP-9 Conjugate was added per well. Following one-hour incubation, the wells were washed and added with 200ul of Substrate Solution to react for 30 minutes. Ultimately, each well was added with 50ul of stop solution, and the optical density was determined with a microplate reader set to $450 \mathrm{~nm}$. 


\subsection{Statistical analyses}

After examining variance equivalence and normal distribution, a nonparametric Mann-Whitney U test was applied to assess the difference between pre-treatment and post-treatment serum concentration of MMP-9, while Chi-squared test was employed to evaluate the correlation between outcomes of patients and clinical characters. Parameters that were statistically significant in the Chi-squared test were further carried out in the binary multiple logistic regression analysis. All analysis was performed with SPSS version 22.0 and the figures were completed with GraphPad version 7.04. A P value $<0.05$ was considered to be significant.

\subsection{Informed Consent}

The protocol was approved by [removed for blind peer review] and in concordance with the Helsinki Declaration. All patients provided written informed consent.

\section{Results}

\subsection{ClinicalCharacteristics of Patients with SSNHL}

A total of 52 patients were enrolled in our study, including $29(55.77 \%)$ male and $23(44.23 \%)$ female, with an average age of 47.12 years (SD:16.93, range 15-76). All patients suffered from unilateral hearing loss, while $84.6 \%$ and $17.3 \%$ of patients accompanied with tinnitus and vertigo, respectively. The left to right ratio was 25:27. The PTA average in patients prior to treatment was $65.07 \mathrm{~dB}$ (SD 27.61, range 10.00-121.50), while the average following treatment turned to $43.22 \mathrm{~dB}$ (SD 29.89, range 2.50-116.25). Based on the initial PTA, 9, 8, 19, and 16 patients were divided into the ascending, descending, horizontal and profound group, respectively. And refer to their final PTA, 20 patients got NR, whereas 7, 14, 11 patients had CR, MI, PI, respectively. (data shown in Table. II)

\subsection{Changes of serum MMP-9 concentration prior to and following treatment in different types of SSNHL.}

Patients with SSNHL had a higher concentration of MMP-9 following treatment as compared with priortreatment concentration, but it was not statistically significant $(116.56 \pm 50.01 \mathrm{ng} / \mathrm{ml}$ vs $131.45 \pm 45.33$ $\mathrm{ng} / \mathrm{ml}, \mathrm{p}=0.054)$. Next, we compared the mean prior-treatment serum concentration of MMP-9 with posttreatment concentration in ascending, descending, horizontal, and profound group. (prior to treatment vs post-treatment, $99.76 \pm 23.44 \mathrm{ng} / \mathrm{ml}$ vs $125.82 \pm 35.50 \mathrm{ng} / \mathrm{ml}, 129.20 \pm 58.33 \mathrm{ng} / \mathrm{ml}$ vs $148.91 \pm 66.43 \mathrm{ng} / \mathrm{ml}$, $112.42 \pm 66.59 \mathrm{ng} / \mathrm{ml}$ vs $128.65 \pm 45.13 \mathrm{ng} / \mathrm{ml}$ and $124.69 \pm 30.95 \mathrm{ng} / \mathrm{ml}$ vs $129.20 \pm 43.01 \mathrm{ng} / \mathrm{ml}$, respectively) .An increase was found in all four groups, however, none of them had statistical significance (Fig.I, $\mathrm{P}=0.094$, $\mathrm{P}=0.574, \mathrm{P}=0.191, \mathrm{P}=0.867$, respectively).

\subsection{Changes of serum MMP-9 concentration prior to and following treatment in different} outcomes of patients with SSNHL.

Given an increase of MMP-9 concentration in patients following treatment was identified, we further detected whether changes in MMP-9 concentration had a significant difference in the outcomes of patients. In treatment responders, mean MMP-9 concentration was elevated from $106.85+-41.40 \mathrm{ng} / \mathrm{ml}$ to $144.03+-$ $37.65 \mathrm{ng} / \mathrm{ml}$ following treatment, while it was decreased from $132.09+-59.21 \mathrm{ng} / \mathrm{ml}$ to $106.82+-49.93 \mathrm{ng} / \mathrm{ml}$ in non-responders. Anecdotally, a difference was found in responders $\left(\mathrm{P}_{j} 0.001\right)$, however, the decrease of MMP-9 concentration in non-responders did not have statistically difference $(\mathrm{P}=0.142)$. Next, we examined if differences existed in each subgroup of treatment responders. Patients in PI, MI and CR subgroup all gained an increase of MMP-9 concentration following treatment (pre-treatment vs post-treatment, 115.62+$40.52 \mathrm{ng} / \mathrm{ml}$ vs $161.23+-44.95 \mathrm{ng} / \mathrm{ml}, 98.45+-49.84 \mathrm{ng} / \mathrm{ml}$ vs $138.46+-32.34 \mathrm{ng} / \mathrm{ml}, 109.87+-20.47 \mathrm{ng} / \mathrm{ml}$ vs $140.99+-25.72 \mathrm{ng} / \mathrm{ml}$, respectively). And with the nonparametric Mann-Whitney U test, we found significant differences in each group (Fig. II, $\mathrm{P}=0.047, \mathrm{P}=0.005$, and $\mathrm{P}=0.038$, respectively).

\subsection{Correlation between outcomes of patients with SSNHL and Clinical characteristics}

Based on the final PTA, patients were dichotomized into treatment responders and treatment non-responders. 
A chi-squared test was performed to disclose the potential correlation between clinical characteristics and outcomes of patients. As indicated in Table. III, patients with increased concentration of MMP-9 following treatment were more frequently accompanied by favorable outcomes $\left(\mathrm{P}=0.007, \chi^{2}=7.436\right)$. However, the incidence of tinnitus or vertigo had no significant difference between the two groups (tinnitus vs vertigo, $\mathrm{P}=0.332$ vs $\mathrm{P}=0.239, \chi^{2}=0.724$ vs $\chi^{2}=1.213$ ). Moreover, treatment responders had similar trends in gender or side of hearing loss as compared with treatment non-responders (gender vs side, $\mathrm{P}=0.353$ vs $\mathrm{P}=0.474$, $\chi^{2}=0.439$ vs $\chi^{2}=0.123$ ). Whereas, patients with favorable outcome was younger than those suffered from treatment resistance $\left(\mathrm{P}=0.03, \chi^{2}=4.623\right)$.

Though the Chi-squared test did not reveal the incidence of tinnitus or vertigo was related with the outcomes, we still put them into binary multiple logistic regression analysis, given several pieces of literature reported the opposite results. Interestingly, the result disclosed changes in MMP-9 concentration was the only factor closely associated with favorable outcomes $(\mathrm{P}=0.008$, $\mathrm{OR}=5.13,95 \% \mathrm{CI}: 1.53-17.28$, Table. III).

\section{Discussion}

To the best of our knowledge, this is the first study about the correlation between changes of MMP-9 concentration and outcomes of patients with SSNHL. In our previous study, we found the concentration of MMP-9 was decreased in patients with SSNHL, as compared with in healthy controls, and the difference had no correlation with the types of SSNHL [12]. The same conclusion is formulated in this further study. Though in these 4 types of SSNHL, the mean concentration of MMP-9 seemed to be elevated following treatment, the increment did not have a significant difference. Besides, this increment seemed more due to the variational therapy responses in different types of SSNHL. Patients with a profound type of SSNHL are known to accompany with the worse outcomes, while patients with ascending type, the prognoses are in contrast. Hence, the post-treatment concentrations of MMP-9 in patients with profound type were almost the same as the pre-treatment concentrations, whereas patients with an ascending type of SSNHL gained significant increment in MMP-9 concentrations following treatment.

The correlations between age, vertigo, or tinnitus with the favorable outcomes of patients with SSNHL are in debate [14-16]. In our study, we displayed that age and the increment of MMP-9 concentration following treatment were correlated to the hearing gain by the Chi-squared test. However, age failed to show the association with outcome in the binary multiple logistic regression test and the increment of MMP-9 was the only factor closely related to favorable outcomes. MMP-9 is recognized as a metalloproteinase to degrade the extracellular matrix and to induce the infiltration of inflammatory cells [17]. Recently, several studies have shown that hyperhomocysteinemia was associated with SSNHL and can induce an increase in the cochlear expression of MMP-2 and MMP-9 in mice models [18-19]. In consideration of these studies, we speculated that MMP-9 might deposit in different sites of the inner ear and induce inflammation by degrading the extracellular matrix. When the damage occurred at the top inner hair cells of the cochlea, patients suffered from ascending type of SSNHL, whereas the damage occurred at the bottom inner hair cells of the cochlea, patients developed descending type of SSNHL. Following treatment, especially with the glucocorticoid, the deposition of MMP-9 in the inner ear decreased and then induced the relief of the inflammatory reaction in the inner ear. Due to this nonspecific deposit, the outcome of patients had no significant difference with the types of SSNHL but with the changes in the concentration of MMP-9 following therapy.

The etiology of SSNHL is still unknown. The immune disorder is considered as one of the major causes of SSNHL, but there is no direct evidence to prove it. Several studies have revealed the correlations between MMP-9 and other diseases. Robaka, et al found serum MMP-9 level was decreased in patients with active SLE, and the level was elevated when SLE turned to an inactive state [20]. Vira H, et al obtained the same conclusion with detecting the polymorphisms of MMP-9 in patients with SLE [21]. In contrast, studies in cardiovascular diseases or tumors indicated that serum conc-entration of MMP-9 was positively associated with the activity of the diseases $[22,23]$. In our study, the altering level of MMP-9 was accordant with the changes in patients with autoimmune disease [24], thus provided further evidence that autoimmune disorder was one of the major causes of SSNHL. 
Altogether, we identified that elevated serum concentrations of MMP-9 was not associated with different types of SSNHL, stratified by the different shapes of the audiogram, but closely correlated to favorable outcomes in patients with SSNHL, therefore it might serve as a prognosis prediction factors of patients with SSNHL. Given the increment of MMP-9 concentration following treatment was accordant with patients with the auto-immune diseases rather than patients with cardiovascular diseases or tumors, it proved another indirect evidence that immune disorder was one of the major pathogenesis in SSNHL.

Limitations in our study are mainly the small case number of patients with SSNHL. We ascribed this limitation to several patients admitted to our hospital with treatment in outpatient department or other hospitals. Whereas, there were still several patients refused to take audiometry test following treatment, which leaves the clinical characteristics uncompleted. Furthermore, we only performed an ELISA test to provide the indirectly evidence, the mechanism of the altering MMP-9 concentration in patients with SSNHL needed to be further proved.

\section{Conclusions}

Our study revealed that elevated serum concentration of MMP-9 following treatment in patients with SSNHL was closely correlated with favorable outcomes. The result is accordant with patients with auto-immune diseases, therefore to provide an indirectly evidence that immune disorder was one of the main pathogenesis in SSNHL. Further study was in need to investigate the underlying mechanism in the increment of MMP-9 concentration following treatment with patients benefited from hearing gained.

\section{Conflicts of interest}

The authors declared there are no conflicts of interest in this work.

\section{Reference}

1. Dicuonzo F, Purciariello S, De Marco A, et al. MR evaluation of encephalic leukoaraiosis in sudden sensorineural hearing loss (SSNHL) patients. Neurol Sci. 2019 Feb;40(2):357-362.

2. Wu CS, Lin HC, Chao PZ. Sudden sensorineural hearing loss: evidence from Taiwan. Audiol Neurootol 2006; 11:151-6.

3. Teranishi M, Katayama N, Uchida Y, Tominaga M, Nakashima T. Thirty-year trends in sudden deafness from four nationwide epidemiological surveys in Japan. Acta Otolaryngol 2007;127:1259-65.

4. Merchant SN, Durand ML, Adams JC. Sudden deafness: is it viral? ORL J Otorhinolaryngol Relat Spec 2008; 70:52-60.

5. Ryan AF, Harris JP, Keithley EM. Immune-mediated hearing loss: basic mechanisms and options for therapy. Acta Otolaryngol Suppl 2002; 548:38-43.

6. De Stefano ME, Herrero MT. The multifaceted role of metalloproteinases in physiological and pathological conditions in embryonic and adult brains. Prog Neurobiol. 2017 Aug; 155:36-56.

7. Chen R, Cai X, Liu J, Bai B, Li X. Sphingosine 1-phosphate promotes mesenchymal stem cell-mediated cardioprotection against myocardial infarction via ERK1/2-MMP-9 and Akt signaling axis. Life Sci. 2018 Dec 15; 215:31-42.

8. Oku T, Shimada K, Kenmotsu H, et al. Stimulation of Peritoneal Mesothelial Cells to Secrete Matrix Metalloproteinase-9 (MMP-9) by TNF- $\alpha$ : A Role in the Invasion of Gastric Carcinoma Cells. Int J Mol Sci. 2018 Dec 9;19(12).

9. Liu Y, Tie N, Bai L. Serum Levels of MDC and MMP-9 and the Relationship Between Serum Levels and Disease Activity in the Patients with Systemic Lupus Erythematosus. Pak J Med Sci. 2015 Jul-Aug;31(4):803-6

10. Jönsson S, Lundberg AK, Jonasson L. Overexpression of MMP-9 and its inhibitors in blood mononuclear cells after myocardial infarction-is it associated with depressive symptomatology? PLoS One. 2014 Aug 25;9(8):e105572

11. Stanciu AE, Zamfir-Chiru-Anton A, Stanciu MM, Popescu CR, Gheorghe DC. Serum Level of Matrix Metalloproteinase-9 in Patients with Head and Neck Squamous Cell Carcinoma. Clin Lab. 2016 Aug 1;62(8):1569-1574. 
12. [removed for blind peer review]

13. Chinese Guidelines for the diagnosis and treatment of Sudden Sensorineural Hearing loss(2015). Chinese Journal of Otorhinolaryngology Head and Neck Surgery,2015(6):443-447.

14. Chung JH, Cho SH, Jeong JH, Park CW, Lee SH. Multivariate analysis of prognostic factors for idiopathic sudden sensorineural hearing loss in children. Laryngoscope . 2015;125(9):2209-2215. doi:10.1002/lary.25196

15. Kim JY, Han JJ, Sunwoo WS, et al. Sudden sensorineural hearing loss in children and adolescents: Clinical characteristics and age-related prognosis. Auris Nasus Larynx . 2018;45(3):447-455. doi:10.1016/j.anl.2017.08.010

16. Kuhn M, Heman-Ackah SE, Shaikh JA, Roehm PC. Sudden sensorineural hearing loss: a review of diagnosis, treatment, and prognosis. Trends Amplif . 2011;15(3):91-105. doi:10.1177/1084713811408349

17. Choi YC, Daiakas MC. Expression of matrix metalloproteinases in the muscie of patients with inflammatory myopathies[J]. Neuroiogy, 2O00.54(1):65_71.

18. Marcucci R, Alessandrello Liotta A, Cellai AP, et al. Cardiovascular and thrombophilic risk factors for idiopathic sudden sensorineural hearing loss. J Thromb Haemost. 2005 May;3(5):929-34.

19. Kundu S, Tyagi N, Sen U, Tyagi SC. Matrix imbalance by inducing expression of metalloproteinase and oxidative stress in cochlea of hyperhomocysteinemic mice. Mol Cell Biochem. 2009 Dec;332(12):215-24.

20. Robak E, Wierzbowska A, Chmiela M, et al. Circulating Total and Active Metalloproteinase-9 and Tissue Inhibitor of Metalloproteinases-1 in Patients with Systemic Lupus Erythomatosus[J]. Mediators of Inflammation. 2006;2006(1):17898.

21. Vira H, Pradhan V, Umare V, et al. Role of polymorphisms in MMP-9 and TIMP-1 as biomarkers for susceptibility to systemic lupus erythematosus patients. Biomark Med. 2019 Jan;13(1):33-43.

22. Opstad TB, Arnesen H, Pettersen AA, Seljeflot I. The MMP-9 -1562 C/T polymorphism in the presence of metabolic syndrome increases the risk of clinical events in patients with coronary artery disease. PLoS One. 2014;9(9):e106816. Published 2014 Sep 5. doi:10.1371/journal.pone.0106816

23. Thammineni KL, Thakur GK, Kaur N, Banerjee BD. Significance of MMP-9 and VEGF-C expression in North Indian women with breast cancer diagnosis. Mol Cell Biochem. 2019;457(1-2):93-103. doi:10.1007/s11010-019-03515-w

24. Matache C, Stefanescu M, Dragomir C, et al. Matrix metalloproteinase-9 and its natural inhibitor TIMP-1 expressed or secreted by peripheral blood mononuclear cells from patients with systemic lupus erythematosus. Journal of Autoimmunity . 2003;20(4):323-331

\section{Hosted file}

Figures.docx available at https://authorea.com/users/335633/articles/461484-evidence-ofimmune-disorder-served-as-the-main-pathogenesis-in-ssnhl-elevated-serum-concentrationof-mmp-9-following-treatment-was-associated-with-favorable-outcomes-in-patients

\section{Hosted file}

Tables.docx available at https://authorea.com/users/335633/articles/461484-evidence-ofimmune-disorder-served-as-the-main-pathogenesis-in-ssnhl-elevated-serum-concentrationof-mmp-9-following-treatment-was-associated-with-favorable-outcomes-in-patients 